\section{Triple terapia antitrombótica en práctica real: utilidad pronóstica de la escala HAS-BLED}

\section{Triple antithrombotic therapy in clinical practice: prognostic utility of HAS-BLED score}

\section{Sr. Editor:}

La prescripción de triple terapia antitrombótica, definida como el uso combinado de ácido acetilsalicílico, un inhibidor de $\mathrm{P}_{2} \mathrm{Y}_{12}$ y anticoagulación oral, está indicada cuando coexisten enfermedades como la cardiopatía isquémica, la fibrilación auricular o la cirugía valvular cardiaca, y supone un reto clínico de prevalencia creciente todavía sin resolver. Debido al incremento en el riesgo hemorrágico, se recomienda que la duración de la triple terapia antitrombótica sea la menor posible, con un máximo período de un año en pacientes con infarto agudo de miocardio e indicación de anticoagulación oral. Otras medidas de consenso incluyen el uso preferente de antagonistas de la vitamina $\mathrm{K}$ para la anticoagulación oral (o bien dabigatrán $110 \mathrm{mg}$ ) y clopidogrel como antiagregante dual (evitando el uso de prasugrel y ticagrelor), la protección gástrica con inhibidores de la bomba de protones y un INR objetivo entre 2,0 y $2,5^{1}$.

La indicación de triple terapia antitrombótica debe establecerse, por tanto, tras la evaluación individualizada del paciente atendiendo a la presencia o ausencia de síndrome coronario agudo, el tipo de stent implantado y el riesgo hemorrágico. Así, en la valoración de este riesgo, la escala HAS-BLED puede ser útil, si bien su trascendencia pronóstica en este contexto clínico y en situación de práctica real han sido poco estudiadas.

Por ello, nos planteamos un estudio de cohortes retrospectivo de pacientes ingresados en cardiología desde enero de 2011 hasta enero de 2015 con prescripción de triple terapia antitrombótica al alta, analizando en la evolución la seguridad del tratamiento en función de la puntuación obtenida en el score HAS-BLED. Se consideraron como eventos clínicos en el seguimiento la mortalidad total, el ictus hemorrágico y la incidencia de sangrado mayor, definido como aquella hemorragia clínicamente significativa que requiere transfusión de al menos dos unidades de hematíes, que precisa ingreso hospitalario, o que causa la muerte.
De 3.781 ingresos consecutivos se identificaron 109 pacientes con triple terapia antitrombótica al alta (edad media $73 \pm 11$ años). El motivo mayoritario para la instauración de dicha terapia fue el diagnóstico de síndrome coronario agudo en pacientes con anticoagulación oral previa (un $83 \%$ de los pacientes por antecedentes de fibrilación auricular). Los pacientes con HAS-BLED $\geq 3$ eran significativamente mayores $(77,6 \pm 7,0$ vs. $68,3 \pm 12,6$ años) y presentaron cifras menores de hemoglobina durante el ingreso $(12,7 \pm 1,6$ vs. $13,5 \pm 1,9 \mathrm{~g} / \mathrm{dL})$, siendo comparables en el resto de variables clínicas y analíticas. Este grupo de pacientes con mayor riesgo hemorrágico (puntuación media del score HASBLED: $3,19 \pm 0,2$ vs. $1,76 \pm 0,4, p<0,001)$, presentó también mayor riesgo trombótico al alta (puntuación $\mathrm{CHA}_{2} \mathrm{DS}_{2}$-VASc: $4,55 \pm 0,9$ vs. $3,67 \pm 1,4, p<0,001)$.

Durante un seguimiento medio de diez meses, se observó mayor incidencia del evento combinado y mortalidad total en el grupo de pacientes con HAS-BLED $\geq 3$ (40\% vs. $24 \%$, y $27 \%$ vs. $11 \%$ respectivamente, $p<0,001)$. En la figura 1 aparece la curva de supervivencia para el evento combinado. No se hallaron diferencias significativas en la incidencia de ictus hemorrágico y sangrado mayor.

Las guías europeas recomiendan la prescripción de triple terapia antitrombótica en pacientes con fibrilación auricular que se someten a intervencionismo percutáneo. Sin embargo, tras la publicación del estudio WOEST ${ }^{2}$, que demostró una reducción de más del $50 \%$ en los eventos hemorrágicos a un año en el grupo de pacientes tratados con clopidogrel y antagonistas de la vitamina $\mathrm{K}$ respecto a aquellos tratados con triple terapia antitrombótica, las últimas guías americanas ${ }^{3}$ ya han incluido la doble terapia (clopidogrel más antagonistas de la vitamina K) como recomendación Ilb. Aunque este estudio presentaba muchas limitaciones, como el reducido tamaño muestral $(n=573)$, el efecto en los eventos de las hemorragias menores, el bajo uso de protección gástrica (37\%) o el uso infrecuente de acceso radial (26\%) fue el primero en demostrar no sólo que la terapia dual presentaba menor tasa de eventos hemorrágicos que la triple, sino que además no era inferior en términos de eficacia. Estos resultados se refrendaron en un amplio registro en práctica real (con más de 12.000 pacientes) ${ }^{4}$, que concluía que la combinación de antagonistas de la vitamina $\mathrm{K}$ y clopidogrel es suficiente para reducir el riesgo de eventos trombóticos tras un intervencionismo coronario. Con esto, parece ser que la adición de Aspirina en este contexto no genera efectos beneficiosos y sí puede incrementar 


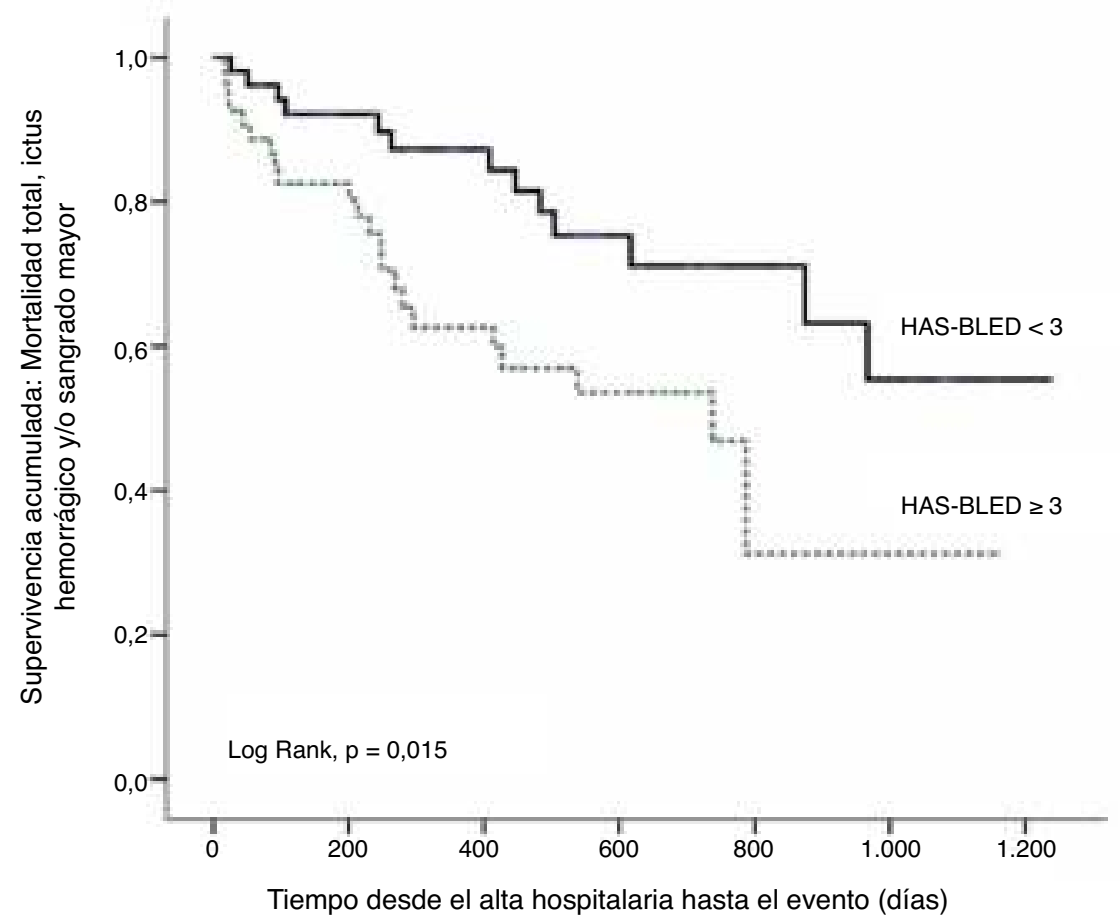

\begin{tabular}{|l|c|c|c|c|}
\hline & $\begin{array}{c}\text { Todos } \\
(\mathrm{n}=109)\end{array}$ & $\begin{array}{c}\text { HAS-BLED }<3 \\
(\mathrm{n}=54)\end{array}$ & $\begin{array}{c}\text { HAS-BLED } \geq 3 \\
(\mathrm{n}=55)\end{array}$ & $\mathrm{P}$ \\
\hline Eventos durante el seguimiento \\
\hline Evento combinado, $\mathrm{n}(\%)$ & $35(34)$ & $13(24)$ & $22(40)$ & $<0,001$ \\
\hline Mortalidad total, $\mathrm{n}(\%)$ & $21(19)$ & $6(11)$ & $15(27)$ & $<0,001$ \\
\hline Ictus hemorrágico, $\mathrm{n}(\%)$ & $2(2)$ & $1(12)$ & $1(2)$ & 0,99 \\
\hline Hemorragia mayor, $\mathrm{n}(\%)$ & $20(18)$ & $8(15)$ & $12(22)$ & 0,34 \\
\hline
\end{tabular}

Figura 1 Eventos adversos durante el seguimiento según la puntuación obtenida en la escala de riesgo hemorrágico HAS-BLED. Curva de Kaplan-Meier para la incidencia del evento combinado durante el seguimiento: mortalidad total, ictus hemorrágico y hemorragia mayor.

la incidencia de sangrado en pacientes de alto riesgo. Así, la escala HAS-BLED podría ser una herramienta útil en la detección de estos pacientes en quienes la terapia dual podría considerarse una alternativa terapéutica de inicio.

Se asumen las limitaciones de un registro unicéntrico y retrospectivo, y el análisis del seguimiento desde una historia médica electrónica, que puede generar pérdida de eventos en el registro. Sin embargo, las conclusiones del trabajo sugieren la utilidad pronóstica de la escala HAS-BLED en pacientes previamente hospitalizados en cardiología e indicación de triple terapia antitrombótica.

\section{Bibliografía}

1. Sorensen R, Gislason G. Tratamiento antitrombótico combinado triple: arriesgado pero a veces necesario. Rev Esp Cardiol. 2014;67(3):171-5.

2. Dewilde WJ, Oirbans T, Verheugt FW, Kelder JC, De Smet BJ, Herman JP, et al. Use of clopidogrel with or without aspirin in patients taking oral anticoagulant therapy and undergoing percutaneous coronary intervention: an open-label, randomized, controlled trial. Lancet. 2013;381(9872):1107-15.
3. January CT, Wann LS, Alpert JS, Calkins H, Cigarroa JE, Cleveland JC, et al. 2014 AHA/ACC/HRS guideline for the management of patients with atrial fibrillation. J Am Coll Cardiol. 2014;64(21):e1-76.

4. Lamberts M, Gislason GH, Olesen JB, Kristensen SL, Schjerming AM, Mikkelsen A, et al. Oral anticoagulation and antiplatelets in atrial fibrillation patients after myocardial infarction and coronary intervention. J Am Coll Cardiol. 2013;62(11): 981-9.

Andrés Cubillos-Arango ${ }^{a}$, Óscar Fabregat-Andrés ${ }^{\mathrm{a}, \mathrm{b}, *}$, Lorenzo Fácila ${ }^{a}$ y Salvador Morell ${ }^{a}$

a Servicio de Cardiología, Consorcio Hospital General Universitario de Valencia, Valencia, España

${ }^{\mathrm{b}}$ Fundación para la Investigación, Hospital General Universitario de Valencia, Valencia, España

* Autor para correspondencia.

Correo electrónico: osfabregat@gmail.com

(Ó. Fabregat-Andrés). 